British Journal of Cancer (20I4) II 0, 3012-3020

www.bjcancer.com

\title{
Referee Acknowledgement for 2013
}

In this issue, we publish the names of those who reviewed manuscripts for us in 2013.

The Editor-in-Chief, Specialist Editors and everyone involved in publishing BJC would like to extend our sincere thanks to them for contributing their expertise and time. Our referees play an invaluable role ensuring that $B J C$ continues to publish the high quality original papers and reviews that make it one of the world's leading oncology journals.

Lauri A Aaltonen

Mieke Josepha Aarts

JP Abastado

JC Abayomi

E Jason Abel

Fritz Aberger

Michael Abern

Ghassan K Abou-Alfa

Robert Abouassaly

A Aboussekhra

Maysa Abu-Khalaf

Anya Adair

Liana Adam

Soheir Adam

Richard Adams

Douglas James Alexander Adamson

Antoine Adenis

Shoaib Afzal

Ilir Agalliu

BB Aggarwal

Manish K Aghi

Nishant Agrawa

Mark Agulnik

Thomas P Ahern

Anders Ahlbom

Munaza Ahmed

Driss Ait Ouakrim

M Aizawa

Jaffer A Ajani

Suminori Akiba

Cem Akin

Olof Akre

Nikolaos Akrivos

Ala-Eddin Al Moustafa

Monther Al-Alwan

Salah-Eddin Al-Batran

Fahd Al-Mulla

Tommy Alain

Joan Albanell

Demetrius Albanes

Paul R Albert

Peter C Albertsen

Laurence Albiges

Derek Alderson

Krasimira Aleksandrova

Ramon Alemany

Jose Enrique Ales-Martinez

Anton Alexandroff

Michalis Alexis

Alexandra Alexopoulou

Raghib Ali

Raza Ali

Shabbir Alibhai

Malcolm R Alison

Alison L Allan

James Allan

Paola Allavena

Martin S Allen-Auerbach

Heike Allgayer

Yves Allory

R Almeida

Gianfranco Alpini

Lucia Altucci

Angeles Alvares-Secord

Secord A Alvarez

Ayesha Alvero

Frederic Amant

James Amatruda
Ziv Amir

Christopher I Amos

Ming-Wen An

Shrikant Anant

Dimitris Anastassiou

Bogi Andersen

Annie Anderson

Karen Anderson

Kristin E Anderson

Therese M-L Andersson

Fabrice Andre

Angeline S Andrew

$\mathrm{H}$ Jervoise N Andreyev

Bernie Andruss

CW Ang

Joo Ern Ang

KK Ang

Takashi Angata

Helen K Angell

Alan Anthoney

Jose Leopoldo Antunes

Thomas Aparicio

Marc Arbyn

Alexandre Arcaro

Caroline D Archer

Jo A Archer

Hannah Arem

Pablo Argueso

Andreas Argyriou

Hendrik-Tobias Arkenau

James Armitage

Terri S Armstrong

Monica Arnedos

Lisen Arnheim-Dahlstrom

Dirk Arnold

Rudolf Arnold

Manuel Arroyo-Morales

Banu Arun

Albert Asante

Margaret Ashcroft

Robert Ashford

George Astrakianakis

M Hammad Ather

Wendy S Atkin

Bradley J Atkinson

Gerhardt Attard

Michaela Aubele

Riccardo A Audisio

Rebecca Auer

Melissa A Austin

Anssi Auvinen

Matias A Avila

Christopher S Awtrey

Daniel Ayude

Basem Azab

Hideo Baba

Sadegh Babashah

Gildy Babiera

Leon A Bach

Thomas Bachelot

Jonathan M Backer

Sunil Badve

Zsuzsanna Bago-Horvath

Chunxue Bai

Richard Baird

L Bakiri

Marije Bakker

Jamie N Bakkum-Gamez
Arjun Balar

Alessandra Balduzzi

Marija Balic

Fran Balkwill

Fatima Baltazar

Cynthia Bamdad

Aristotle Bamias

Udai Banerji

Maggi Banning

Bo-Ying Bao

Ying Bao

Gil Bar-Sela

Mariagnese Barbera

NV Bardakos

Nathalie Bardin

Christopher A Barker

Elizabeth Barley

Jill S Barnholtz-Sloan

$\mathrm{R}$ Baron

Valeria Barresi

J Bartek

Harry Bartelink

Steven Barthel

John MS Bartlett

Rupert Bartsch

Morteza Bashash

E Bastiaannet

Bristi Basu

Luc Bauchet

Eric Baudin

Robert C Baxter

Boon-Huat Bay

Neil Bayman

Ian LP Beales

Harry D Bear

Michel Beaugrand

Jens Bedke

Tomasz M Bee

Axel Behrens

Christian Beisland

Abbes Belkhiri

Christopher Bell

Diana Bell

Cecelia A Bellcross

Mattias Belting

Offir Ben-Ishay

Sergei Benavente

Manuel Benavides

CA Benbassat

Leonidas Benetatos

Maria Benevolo

Charles L Bennett

Kathleen Bennett

Stephane Benoist

Wendie A Berg

Christine Bergeron

Thierry Berghmans

Roberto Bernabei

Phillip S Bernard

Rene Bernards

Massimiliano Berretta

Amy Berrington de Gonzalez

Alfredo Berruti

Giovanni Nicolao Berta

Giulia Bertolini

Francesco Bertoni

Marianne Berwick

Benjamin Besse
Marc Besselink

Chetan Bettegowda

Mark Bettington

Charlotte Bevan

Rohit Bhargava

Rupal S Bhatt

Sudin Bhattacharya

Ettore Biagi

Claudio Bianchi

Giampaolo Bianchini

Roberto Bianco

Ettore Bidoli

Stefan Bielack

Claudia Bighin

Lucinda Billingham

Daniel Birnbaum

Michael J Birrer

John Francis Bithell

Georg A Bjarnason

Esther P Black

Sarah P Blagden

Giovanni Blandino

Roger C Blanks

Jane M Blazeby

Harry Bleiberg

Maria Blettner

Karen Block

Elisabeth Bloemen

Guido Bocci

Stefania Boccia

Christoph Bock

Alan V Boddy

Barrie Bode

Katja Boehm

CF Boerkoel

Paolo Boffetta

Deborah A Boggs

Elfriede Bollschweiler

Guido Bommer

Benjamin Bonavida

Mary Bond

Melissa L Bondy

Laura Bongiovanni

Corrado Boni

Luca Boni

Mathieu Boniol

Dominique Bonnet

Stefanos Bonovas

Chanchai Boonla

Renato Borges Fagundes

Signe Borgquist

Scott Borinstein

Markus Borner

Jaclyn Lee Fong Bosco

Marcus Bosenberg

Cristina Bosetti

FT Bosman

Tom Boterberg

Donald Bottaro

Jan Bouchal

Etienne Boulter

Liam Bourke

Anne-Marie Bouvier

Mark Bower

Kathleen Boyd

Mark Boyd

Norman F Boyd

Ross W Boyle 
Mats Brannstrom

Boudewijn JM Braakhuis

Sergio Bracarda

Chiara Braconi

Daniel Bradley

Julie R Brahmer

Etienne GC Brain

Kate E Brain

Dejana Braithwaite

Michael Graham Bramble

Simon R Bramhall

Vivien Bramwell

Judith Brand

Klaus Brasso

Hiltrud Brauch

Michael Braun

Caroline A Bray

Karine Breckpot

William Breitbart

Meagan Elizabeth Brennan

Alina Brenner

Hermann Brenner

Walburgis Brenner

Ian Breunig

David $\mathrm{H}$ Brewster

Emilio Bria

John Bridgewater

Oscar Briz

Angela M Brodie

Bertha Brodin

Massimo Broggini

John Brognard

Sabine Brookman-May

Deborah M Brown

Elizabeth Brown

Ewan Brown

Gina Brown

Janet E Brown

Jennifer Brown

Justin C Brown

Karen Brown

Kristy Brown

Linda F Brown

Robert Brown

Adam Brufsky

Skjalg Bruheim

Jordi Bruix

Matteo Brunelli

Antonella Brunello

Andrea Brunner

Valerie G Brunton

Nele Brusselaers

Odd Terje Brustugun

Christina M Buchanan

Tomas Buchler

Donald J Buchsbaum

Alessandro Buda

Lars Budaus

Reinhard Buettner

Jack D Bui

MEC Buim

Alessandra Buja

Ronald M Bukowski

Cynthia M Bulik

Jean-Luc Bulliard

Joy Marilyn Burchell

Maximilian Burger

JA Burgers

Alan Burnett

Howard A Burris

John Butler

Lesley M Butler

Alison J Butt

Graham Byrnes

Miguel Caballero

Giuseppe Cabibbo

David P Cabone

Hong-Bing Cai

Paolo Fabrizio Caimi

Vittorio Calabrese

Bruno Calabretta

George Calin

Matthew Calister

Jerry Call

Raffaele A Calogero
Lido Calorini

Richard J Calvert

Diego F Calvisi

Alfonso Calvo Gonzalez

Stefano Calza

David Cameron

E Ramsay Camp

Gabriella Campadelli-Fiume

Moray J Campbell

Paul J Campbell

Peter Campbell

Lia Campos

Jordi Camps

Giovanni Camussi

Silvana Canevari

Natasha J Caplen

Federico Cappuzzo

Giorgio Capuano

S Caradonna

Miguel Carballo

Stephanie Heon Cardarella

Vincenzo Cardinale

Elyce Cardonick

Chris R Cardwell

Lisa Carey

J Carles

Joyce Carlson

Robert W Carlson

James Carmody

Piero Carninci

Tania Carreon-Valencia

C Ross Carter

Jonathan Carter

Brett S Carver

Joseph R Carver

Paolo G Casali

Oriol Casanovas

Stefano Cascinu

Donald O Case

Carlos A Casiano

Philippe A Cassier

Elias Castanas

Victoria Castel

Chiara Castelli

$\mathrm{X}$ Castells

Xavier Castells Oliveres

Luciano Castiello

Rogerio M Castilho

Philip Castle

Javier S Castresana

Jane A Cauley

Jamie Cavenagh

Jonathan Cebon

Paloma Cejas

Sebastian Cerdan

James R Cerhan

Martin Cernvall

Andres Cervantes

GO Ceyhan

YK Chae

Anees B Chagpar

Kishore B Challagundla

Anthony J Chalmers

Marc C Chamberlain

Eric Chamot

Anthony TC Chan

Eddie Shu-yin Chan

Jimmy Yu Wai Chan

Dhyan Chandra

Bei-Hung Chang

Cindy M Chang

David Chang

Helena R Chang

PL Chang

Jenny Chang-Claude

Chun Chao

Caroline Chapman

Judith-Anne W Chapman

Alison Chapple

Kittipat Charoenkwan

Etienne Chatelut

Nilanjan Chatterjee

P Chaturvedi

Ian Chau

Nicole G Chau
Mariana Chavez-MacGregor

Neetu Chawla

Beiyun Chen

Brenda Chen

Eric X Chen

Fei Chen

Guo-Lin Chen

Hsiuchen Chen

Jing Chen

Qi Chen

Ruey-Hwa Chen

Wanjun Chen

Wei R Chen

Wendy Y Chen

Yao-Tseng Chen

Yuming Chen

Zhe-Sheng Chen

Zhu Chen

Ann-Lii Cheng

Ching-Lan Cheng

Liang Cheng

Pi-Wan Cheng

Kwok-Leung Cheung

Dennis S Chi

Kim N Chi

Kee Seng Chia

Sarah Chiang

Sum-Fu Chiang

Anna M Chiarell

Tsutomu Chiba

Simion Chiosea

Costel Chirila

Cristine Chisholm

Julia Clare Chisholm

Kenny Chitcholtan

Rowan T Chlebowsk

Nam Hoon Cho

William Cho

Haesun Choi

Kang-Yell Choi

Jeffrey Chou

Soumitra Kumar Choudhuria

Fahmid Chowdhury

Indrajit Chowdhury

Simon Chowdhury

Thoralf Christoffersen

Quincy Chu

Wen-Ming Chu

Michael Chua

Ock K Chuna

Clement T Chung

Doo Hyun Chung

JW Chung

Jinsoo Chung

Maurizio Cianfriglia

Fortunato Ciardiello

Joseph Ciccolini

Ashley Cimino-Matthews

Diana cittelly

Carlos J Ciudad

Peter Clark

Peter E Clark

Christina Clarke

Robert Clarke

Stephen J Clarke

Elizabeth Claus

Henrik Clausen

Jacqueline Clave

Anne-Marie Cleton-Jansen

Alan Coates

Philip J Coates

David Cobrinik

Pierluigi Cocco

Andrew James Cockbain

Hiram S Cody III

Jan Willem Coebergh

Robert J Coffey

Anna E Coghill

Debbie Cohen

Steven J Cohen

Bertrand Coiffier

Graham Colditz

Andrew J Coldman

LA Cole

Robert E Coleman

Rivka Colen

A Dimitrios Colevas

Helen M Coley

Stephane Collaud

Marco Colleoni

Denis Collins

Giuseppe Colloca

Gerolama Condorell

Barbara Conley

Chris Conlon

Roisin M Connolly

Baqiyyah Conway

Gordon Cook

Colin S Cooper

Matthew R Cooperberg

Mhairi Copland

Olivier coquere

Maria Valeria Corrias

Pippa G Corrie

Javier A Cortes

Alessandro Corti

David O Cosgrove

Ender Coskunpinar

Antonio Costanzo

Eithne Costello

Jose Antonio Costoya

GM Cote

M Cotterchio

Bettina Couderc

Viven Coulson-Thomas

Jonathan Coulter

Laurent Counillon

Sarah Coupland

Elisabeth Couto

Allan Covens 
Soumita Das

Piyali Dasgupta

Somenath Datta

Adil I Daud

Sarah E Daugherty

Elizabeth Davies

Julia Davydova

Sarah-Jane Dawson

Fiona L Day

Lukejohn Day

Matthew Day

Enrique de Alava

Johann S de Bono

Filippo de Braud

Remco de Bree

ML De Bruin

John de Caestecker

Marianna de Camargo Cancela

Vittorio de Franciscis

Ugo De Giorgi

Antonio Garcia de Herreros

Harry J de Koning

Ruggero De Maria

Ramon Andrade Bezerra de Mello

Nancy De More

Benigna M de Oliveira

Paolo De Paoli

Theo M de Reijke

Jonas de Souza

Eduardo De Stefani

Hugues de The

Enrico N De Toni

Elly De Vlieghere

Stefan de Vogel

Esther de Vries

Ronald de Wit

Silvia Deaglio

Jason LJ Dearling

Maria Debiec-Rychter

Frederic Debieve

Waldemar Debinski

Banerjee Debrabata

Jessica T DeFrank

Hadassa Degani

Ettore degli Uberti

Amy C Degnim

Linda A deGraffenried

Scott M Dehm

TJA Dekker

Annarosa Del Mistro

Maguy Del Rio

Jesus Del-Mazo

Nikki A Delk

Curt DellaValle

Gianfranco Delle Fave

Isabelle Deltour

Sandra Demaria

Wendy Demark-Wahnefried

Ronald P DeMatteo

Douglas James Demetrick

Pieter Demetter

David Denardo

James W Denham

David T Denhardt

Marc G Denis

Paul Dent

Sharon Y Dent

Ithaar H Derweesh

Elena Deryugina

Jayesh Desa

IME Desar

Teresa L Deshields

Hari A Deshpande

A Dey

Giuseppe Di Lorenzo

Federica Di Nicolantonio

Dolores Di Vizio

Eleftherios P Diamandis

Michael D Diamantidis

Michael Dickinson

Wolfgang Dietmaier

Olaf Dietrich

JJ Dignam

Palma Dileo

Lisa Diller
Mary Dillon

Daniela M Dinulescu

Caroline Diorio

Ahmet Dirican

Luc Yves Dirix

Piet Dirix

Luitpold Valentin Distel

Jurgen Dittmer

Nullin Divecha

Rosa Divella

Michael Dixon

Kahnh Do

Sheila A Doggrell

Luigi Dogliotti

Gilles Dolivet

B Dome

Gemma Dominguez

Charlotte J Dommering

Maria Gabriella Dona

Susan J Done

Valerian Dormoy

Isabel dos Santos Silva

Kenny Douglas

Anneli Marie Dowler Nygaard

Amy Downing

Jerome Doyen

Brendan Doyle

George Dranitsaris

Paul Drew

Jean-Pierre Droz

Mark Drummond

Jozef Drzewoski

KL Du

Andreas du Bois

Louis Dubeau

Thomas Duberge

Purnima Dubey

Anna Dubrovska

Katie Duckworth

Dan G Duda

Jozsef Dudas

Alfonso Duenas-Gonzalez

Matthias Duerst

Florence Duffaud

Stephen W Duffy

Catherine Duggan

Jonathan S Duke-Cohan

Nickolai Dulin

Reinhard Dummer

Marco Durante

Aurelie Dutour

M Duvic

Mark Duxbury

Martin Dyer

Lars Dyrskjot

Alan R Eastman

Wilfried Eberhardt

Lisa M Ebert

Florian Ebner

John Ebos

Andre Eckardt

Iris E Eder

Adrienne L Edkins

Dylan R Edwards

Joanne Edwards

John Edwards

Ros Eeles

Kathleen M Egan

Grace Egeland

W Eiermann

Ram Eitan

Carina Eklund

Martin Eklund

Karin Ekstrom-Smedby

Samir El Mofty

EM El-Omar

Ahmed Elattar

Joanne Watters Elena

Heather Eliassen

Laurie Elit

Elena Elkine

Tony Elliott

Lee M Ellis

Matthew J Ellis

Peter Ellis
Pamela Ellsworth

Anneli Elme

Leisha Ann Emens

Steffen Emmert

Cathy Eng

Eric Engels

Gerda Englholm

Hideki Enokida

Vikki A Entwistle

Mara Epstein

Paul Epstein

Oleg Eremin

Britt K Erickson

Ferry ALM Eskens

David Euhus

DGR Evans

Tracey Evans

Marianne Ewertz

Susanne Fussel

Muller Fabbri

Guy Fagherazzi

Christopher Fairley

Sara Faithfull

Marco Falasca

Gerald Falchook

Stephen J Falk

Lesley Fallowfield

Fang Fang

Fu-Min Fang

Alessandro Fanzani

Francoise Farace

Farris Farassati

Fabio Farinat

Carrie Featherstone

Todd Fehniger

Deb Feldman-Stewart

Jaime Feliu

Ashley S Felix

Liviu Feller

Emanuela Felley-Bosco

Annika Fendler

Volker Fendrich

Bing-Jian Feng

Gong Feng

Ian S Fentiman

Gerardo Ferbeyre

Liesbeth Ferdinande

Cristiano Ferlini

Almudena Fernandez-Briera

Silvia Fernandez de Mattos

Rosalie E Ferner

Gabriella Ferrandina

Robert L Ferris

Charles Ferte

Maria Feychting

Heinz Herbert Fiebig

William Figg

JD Figueroa

Helen Filmore

Christian D Fingas

Sirio Fiorino

PB Fisher

Harold Fisk

Margaret I Fitch

Rebecca Fitzgerald

Patricia Fitzpatrick

Keith T Flaherty

James M Flanagan

Mark T Fleming

Olivia Fletcher

Roberto Flores

Tullio Florio

Gunnar Folprecht

Constantino Fondevila

DB Fontein

Stuart J Forbes

Hugo Ford

James M Ford

Jennifer S Ford

Michele Forman

Matthew Forshaw

Martin Forster

Cristina Fortes

Ciardiello Fortunato

Sophie Dorothea Fossa

Christina Fotopoulou

William D Foulkes

Joe Fox

Simon A Fox

Agnes Francois

Brigitte Franc

Silvia Franceschi

Heather Francis

Wilbur Alan Franklin

Birgitte Lidegaard Frederiksen

Henrik Frederiksen

Irma Fredriksson

Steve J Freedland

Christian Frezza

Zvi G Fridlender

Rafael Fridman

Wolf Herve Fridman

Christine Friedenreich

Nicolaus Friedrichs

Helmut Friess

Thomas Frisell

Lin Fritsch

Martin Fruh

Chuan Gang Fu

Jie $\mathrm{Fu}$

Ota Fuchs

N Fujimoto

Mayumi Fujita

Hiroshi Fukamachi

Simone Fulda

Chunkit Fung

Teresa Fung

Fiona Furlong

Junji Furuse 
Faith Gibson

Rachel J Gibson

Spencer B Gibson

Todd M Gibson

Brenda Hofer Giddings

Gretchen L Gierach

ourik A Gietema

Ethel S Gilbert

Mark R Gilbert

Graham Giles

Ziv Gill

Matthew T Gillespie

Livia Giordano

Elisa Giovannetti

Nicolas Girard

Brendan V Girdler-Brown

Olivier Gires

Ayush Giri

Albert W Girotti

Jacopo Giuliani

Aprile Giuseppe

Julia L Glade-Bender

Paul Glare

Sally Glaser

Rosalind Margaret Glasspool

Hilary Glen

Paul Glen

Bengt Glimelius

Rob Glynne-Jones

Michael Gnant

Helenice Gobbi

Peter Goedegebuure

Shom Goel

Wolfgang Goering

James J Going

Richard Goldberg

I David Goldman

Jonathan W Goldman

Rado Goldman

Torsten Goldmann

Chris Goldring

David Goldstein

Donald Peter Goldstein

Klaus Golka

Lauren Gollahon

Candelaria Gomez-Manzano

Tatsuo Gondo

Zhihong Gong

Andrew Gonzalez

Ana Maria Gonzalez-Angulo

John L Gore

Glenwood D Goss

Noriko Gotoh

Charles Gourley

Tim M Govers

Cristina Gravalos

Heike I Grabsch

Sergio A Gradilone

Ullrich Graeven

Janet Graham

Gordon Grahovac

Matthew J Grainge

Steven Grant

William B Grant

Claudia Gravekamp

Shannon Graver

Peter Gray

Steven G Gray

Valery Z Grdzelishvili

Mel Greaves

Daniel M Green

Edward Greenfield

Tim F Greten

S Michael Griffin

Anita Grigoriadis

Bogdan Grigoriu

Corinne E Griguer

Robert J Grimer

Peter Philipp Grimminger

Pavel Gromov

Patti Groome

Martha A Grootenhuis

Cary P Gross

Francesco Grossi

Ellen K Grov
Franz X Gruber

Thomas Gruenberger

Victor Gruenwald

Richard G Grundy

Michael Grusch

Zhennan Gu

Ng Chong Guan

Valentina Guarneri

Ayca Gucalp

Henk-Jan Guchelaar

Ines Guetgemann

Joel Guigay

C Guillen-Ponce

Severine Guiu

Donald Gullberg

James L Gulley

William J Gullick

Preethi Gunaratne

Michael Dee Gunn

Bin Guo

Li-tao Guo

Lilu Guo

Nancy Lan Guo

Ajay Gupta

Ramneek Gupta

Tejpal Gupta

James G Gurney

Barry A Gusterson

Uwe Guth

Balazs Gyorffy

Matthias Haas

Michael Haas

Nagy Habib

Neville F Hacker

Ulrich Thorsten Hacker

Allan K Hackshaw

Bruce C Haffty

Thorsten Hagemann

Angela Hague

Qin Haide

Pierre Hainaut

Abraham A Hakimi

Ruth Halaban

KJ Halazun

Eric J Hall

Janet Hall

John Hall

Peter S Hall

Daniel G Haller

Florian Haller

Jonathan Ham

Ryuji Hamamoto

Anne W Hamburger

Richard Hamelin

Robert J Hamilton

William T Hamilton

Pascal Hammel

Peter S Hammerman

Hans Hammers

Kwang-Hyub Han

Wonshik Han

Andrew Hanby

Barry W Hancock

Greg Hannigan

Jean-Michel Hannoun-Levi

Johan Hansson

Desiree Hao

Yasuaki Harabuchi

Hideyuki Harada

Masaru Harada

Kamran Harati

Jenny Hardingham

Alexandre Harle

Diane M Harper

Kevin J Harrington

Holly Harris

Robin Harris

Ewen Harrison

Mark Harrison

Sian Harrison

Simon J Harrison

Douglas J Hartmann

R Donald Harvey

Naoki Hashimoto

Manal M Hassan
Elizabeth Hatch

Jari Haukka

Laura J Havrilesky

Douglas S Hawkins

Maria A Hawkins

Michael Max Hawkins

Tania Hawthorn

Shinya Hayam

Daniel F Hayes

John D Hayes

Richard B Hayes

Larry Hayward

Biao $\mathrm{He}$

Jaclyn F Hechtman

David W Hedley

Rakesh Heer

Karen A Heichman

Lene N Heidemann

Volker Heinemann

Laura Heiser

Florian Heitz

I Hellstrom

Kari Hemminki

Paul T Henderson

Alain Hendlisz

Michael Hendryx

Jan G Hengstler

Christophe Hennequin

Claudia I Henschke

Harvey Hensley

Alexander G Heriot

Luis A Herrera

Rolando Herrero

C Simon Herrington

Hermann Hertel

Rodney John Hicks

Ernest Hidalgo

Martin S Highley

Jun Hihara

Allan Hildesheim

Eberhardt Hildt

Mark Hill

Thomas Hillen

Cimona V Hinton

Michelle S Hirsch

Michie Hisada

Tzu-Hung Hisao

Eiso Hiyama

Eva Hnizdo

Susan Hodgson

OS Hoekstra

Silvia Hofer

Karen E Hoffman

Markus Hoffmann

Johannes Hofland

Paul Hofman

Solveig Hofvind

Brigid L Hogan

Pancras CW Hogendoorn

Lieke $\mathrm{Hol}$

Joanna Holbrook

Stefan Holdenrieder

Kevin Hollevoet

Marianne Holm

Christine Holmberg

Lars Holmberg

Christopher Holsinger

$\mathrm{H}$ Hondermarck

Hubert Hondermarck

Friedemann Honecker

David S Hong

Ji-Hong Hong

Jacob P Hoogendam

Regina Hooley

Michael Hopfner

Paul G Horgan

Alan Horwich

Steven Horwitz

H Dean Hosgood

Kent Hoskins

Anthony Howell

Sacha J Howell

Stephen B Howell

Ralph $\mathrm{H}$ Hruban

Jenn-Ren Hsiao

Ching-Yeh Hsiung

Chaosu $\mathrm{Hu}$

Rong-Gui Hu

Cai Huang

Qihong Huang

Michael Hubalek

Rebecca A Hubbard

Peter E Huber

Stephan Huber

Cyrille Huchon

RS Hudson

Anika Huesing

Thomas A Hughes

Kam Hui

Rayjean J Hung

Ted Hupp

SP Hussain

Syed A Hussain

Clara Hwang

Barry Iacopetta

Roberto Iacovelli

Daisuke Ichikawa

Shingo Ichimiya

OK Idowu

Natalia Ignatenko

Hisa Iinuma

Masahide Ikeguchi

Ray Kruse Iles

Marius Ilie

David H Ilson

Yu Imamura

John D Imig

Evgeny Imyanitov

Federico Innocenti

Akira Inoue 
Michael AS Jewett

Jun Yuan Ji

Wei-Hua Jia

Yuan Jian

M Jiang

Yixing Jiang

Long R Jiao

Xiaoli Jiao

J Jin

Ying Jin

Yelena Jinjigian

Sao Jiralerspong

Karin Jirstrom

Moyez Jiwa

Lee JM

Heikki Joensuu

SA Johannesdottir

Christoffer Johansen

Mattias Johansson

Silvia Johansson

Esther John

Thomas John

Nina Fons Johnsen

Jonas T Johnson

Keryn Johnson

George Don Jones

Robert J Jones

Robin L Jones

Terry M Jones

Benedicte Jordan

Hannah C Jordan

V Craig Jordan

Trine Lembrecht Jorgensen

Richard W Joseph

Jingfang Ju

Adrian M Jubb

Rosalyn Juergens

Lucienne Juillerat-Jeanneret

Eva Jungel

Johannes H Kaanders

Linda Kaerlev

Miklos Kalapos

Eniko Kallay

Holger Kalthoff

Farin Kamangar

Ashish Kamat

M Kanat-Pektas

Susan E Kane

Daehee Kang

Gyeong Hoon Kang

Reiji Kannagi

Steven C Kao

Jack H Kaplan

Sara Karami

Christos S Karapetis

Rotem Karni

Naoyuki Kataoka

Santosh K Katiyar

Kiyoko Kato

Masaru Katoh

Elad Katz

Koji Kawakami

Klaus Kayser

Yavuz Kececi

Paul Keeley

Daniel Keizman

Margaret Kelaher

Fergal Kelleher

Mark R Kelley

Robin Katie Kelley

Cathy Kelly

Charles Kelly

Karl T Kelsey

Richard Kennedy

Steve Kennel

Lukas J Kenner

Shannon C Kenney

Matthew Kent

Brent D Kerger

TMA Kerkhofs

Chris Kevil

Timothy J Key

Khandan Keyomarsi

Hanif Khalak

Asif Khalid

\section{A khanna-gupta}

Leeka Kheifets

Joseph D khoury

Asim Khwaja

AK Kiemer

Sharon L Kilbreath

Anne E Kiltie

Hye Jung Kim

Jane J Kim

Ki-Yeol Kim

Nam Kyu Kim

WS Kim

Wun-Jae Kim

Alan Kimber

Ann Dorothy King

Michael R King

Anne Kinhult-Stahlbom

Leo J Kinlen

Yoshikazu Kinoshit

Kathryn M Kinross

Lawrence Kirschner

Cari M Kitahara

Astrid Olsnes Kittang

L Klampfer

Jorg Kleeff

Bernard Klein

Ruth A Kleinerman

Daniel Klevebring

Carolyn Klinge

Burkhard Kneitz

Robert Knobler

Jennifer Knox

Keith L Knutson

Andrew $\mathrm{H}$ Ko

Ying-Chin Ko

Takashi Kobayashi

Inke Koenig

Manolis Kogevinas

Sergio Koifman

Dieuwertje EG Kok

Young-Yun Kong

Raymond L Konger

Ikuo Konishi

Jan J Kootstra

Mladen Korbelik

Marta Korbonits

Chihaya Koriyama

Hasan Korkaya

Greg Korpanty

Takeo Kosaka

Jill Koshiol

K Kostev

Michael I Koukourakis

Anita Koushik

Michal Kovac

Luiz P Kowalski

Christiaan A Krabbe

Sigrid Kraggerud

JR Kramer

M Krause

Stephanie Kreis

Michaela Kreuzer

Rebecca Kristeleit

Glen Kristiansen

Hans Krokan

Mary E Kroll

Carol Kruchko

Sebastian Krug

Arnold Kruse

Frank AE Kruyt

Ja-Lok Ku

Omer Kucuk

Henry Kuerer

Roland P Kuiper

Ernst Kuipers

Gopal C Kundu

Chikara Kunisaki

Min-Liang Kuo

Michael E Kupferman

Silke Kuphal

Peter Kuppen

Razelle Kurzrock

Brian Kushner

A Kwong

Richard G Kyle
Satoru Kyo

Natasha Kyprianou

Robert M Kypta

Stavroula Kyriazi

Caterina AM La Porta

Carlo La Vecchia

Diego J Laderach

Lucie Lafay-Cousin

Guy Lahat

Chiung-Ru Lai

Gabriel Y Lai

Maode Lai

Quirino Lai

Christel B Lajer

Sunil R Lakhani

Enzo Lalli

Elaine Lam

Paul C Lambert

Paul F Lambert

Sylvie D Lambert

Johnathan M Lancaster

David Landau

Lorenza Landi

Sven A Lang

Carol A Lange

Christian M Lange

Scott M Langevin

Ruth E Langley

Cord Langner

Carita Lanner

Donald Lannin

Carlo Largiader

James M Larkin

Timothy L Lash

ilke Lassmann

Eddie Lau

Joseph W-Y Lau

Ulrich Lauer

Dominique Laurier

Carlo LaVecchi

Alessandro Laviano

PS LaViolette

Marialuisa Lavitrano

Malcolm Law

Brian R Lawson

Sigurd Lax

GT Layer

Alexander Lazar

Dung $\mathrm{T}$ Le

Long P Le

Michael Leahy

Andre Lechel

Nicolas Lecomte

Fabrice Lecuru

Anne WM Lee

Chul-Joo Lee

Clara N Lee

Francis Y Lee

Jeannette Y Lee

Jeffrey Lee

Jung Eun Lee

Lukas Jyuhn-Hsiam Lee

Patrick WK Lee

Peter Lee

Yuan-Chin Amy Lee

Jacqueline Lehmann-Che

Thomas Lehnert

Kaisa Lehti

Zhang Lei

Cedric Lemogne

Marc Lenburg

Riccardo Lencioni

Juis Leon Mateos

Candice Lesage

Gregory B Lesinski

Thomas Leto

Ping-Chung Leung

Zohar Levi

Jelena Levitskaya

Sarah Lewis

Stephen Lewis

Joan Lewis-Wambi

Guojun Li

ingmei Li

C-Z Liang

R Liang

D Joshua Liao

GJ Liefers

Eva Lieto

Carol S Lim

Hui-Kuan Lin

Hui-Ming Lin

Jin-Ching Lin

Jou-Wei Lin

Steven $\mathrm{H}$ Lin

Yulan Lin

Yun-Wei Lin

Zhong-Zhe Lin

Amy M Linabery

Claus Lindbjerg Andersen

Annika Lindblom

Gerald P Linette

Mathew Links

Sabine K Linn

Athena Linos

Virginia R Litle

Kate Littin

Mark Peter Little

F Liu

Jason J Liu

Kenneth J Livak

Borje Ljungberg

Lorenzo Lo Muzio

Marc BI Lobbes

Massimo Loda

Fiona Lofts

Richard FA Logan

Susan Logan 
Roberta Maestro

Pamela Magee

Tony Magee

Ronald J Maggiore

Chantal Magne Nde

Siavosh Mahboobi

Haider Mahdi

Enes Makalic

George Makedonas

Keijo Makela

Markus J Makinen

M Malaguarnera

Reza Malekzadeh

Hassan Z Malik

Manzoor Malik

Sreelakshmi Mallappa

Frederick Mallette

Mahitosh Mandal

Laura-Maria S Mandanat

Vincenzo Dario Mandato

Santos Manes

AM Manganon

Janine L Mansi

Wasat Mansoor

David Mant

Alberto Mantovani

Anthony Maraveyas

Robert E Marcus

M Mareel

Vitaly Margulis

Jose JG Marin

Camille Maringe

Christos Markopoulos

Athina N Markou

Svetomir N Markovic

Joseph Markowitz

Maria Marples

Sharon Marsh

Paloma Martin-Sanz

Alberto M Martelli

Lesley-Ann Martin

Anna Martling

Tsutomu Masaki

Giovanna Masci

Luca Mascitelli

Gianluca Masi

Robert Mason

Gita V Massey

Giuseppe Masucci

Naoya Masumori

Daniela Matei

Paul Mathew

John Mathews

Junichi Matsubara

William Matsui

Andrea Mattevi

Katherine Matthay

Mirjami M Mattila

Julie A Mattison

Barry H Maurer

Dimitris Mavroudis

Astrid Mayer

Deborah K Mayer

Erika L Mayer

Musa Mayer

Alan Maynard

Tapati Mazumdar

Colleen McBride

William H McBride

Catherine McCabe

Susan E McCann

Annemarie McCarthy

Colleen M McCarthy

James McCaul

Tim McCavit

David J McConkey

Peter McCulloch

Lauren McCullough

Marjorie L McCullough

Jasmine A McDonald

Archana J McEligot

John McGregor

Colin McKay

Pamela McKay

Rana McKay
Mark McKeage

Catriona McLean

Donald McLeod

Jordana McLoone

Andrew McMillan

Donald C McMillan

Richard JQ McNally

Iain A McNeish

Sean McPhail

Janice M Mehnert

Ranee Mehra

Alan A Melcher

Sebastian Melchior

Ignacio Melero

Bohuslav Melichar

Beatrice Melin

Davide Melisi

Joseph Menczer

Tehillah Menes

Christine Menetrier-Caux

Maxwell V Meng

Alexander M Menzies

MA Merritt

Melissa A Merritt

Wilma E Mesker

Leonid Metelitsa

Kerstin meyer

Tim Meyer

Jeffrey A Meyerhardt

Michael Z Michael

Martin Michaelis

Dominique S Michaud

Evangelos D Michelakis

Carine Michiels

T Michikawa

Mark R Middleton

Yoshio Miki

Michael Milano

Andrea Milbourne

Michele Milella

Anthony B Miller

Joshua Miller

Michael Millward

Daniele Minardi

Tommaso Claudio Mineo

Chen Mingwu

Giuseppe Minniti

Bruce D Minsky

Ollie Minton

Harald Mischak

W Keith Miskimins

Gillian Mitchell

Cathy Mitchelmore

Anirban P Mitra

Tetsuya Mitsudomi

Martina Mittlboeck

Hideaki Miyake

Masayuki Miyake

Yin Yuan Mo

Francesmary Modugno

Christine Moelzer

Samuel C Mok

Tony SK Mok

David R Mole

L Rhoda Molife

Henrik Moller

Luca Mologni

Alison M Mondu

Silvio Monfardini

Bradley J Monk

Justin Monnier

JRT Monson

Jean-Jacques Monsuez

G Monteith

Maurizio Montella

Michael Montemurro

Juan Carlos Montero

Maria Montoya

David H Moore

Kathleen N Moore

Patrick S Moore

Richard G Moore

Ronald B Moore

Anthony Moran

Jerome Moreaux
Jan S Moreb

Miriam C Morey

Bruno Morgan

Maria P Morgan

Todd M Morgan

Daniel Morgensztern

Giuseppe Morgia

Richard Moriggl

Ryan Morin

Chigusa Morizane

David Morris

Eva Judith Ann Morris

Melanie Morris

Tiffany Morris

audrey morrison

Helen Morrison

Cynthia C Morton

Dion G Morton

Gerard C Morton

Lindsay M Morton

Kath A Moser

Richard Moser

KA Moses

Chaya Moskowitz

Sue M Moss

Bianca Moster

Marcella Mottolese

Lina $\mathrm{Mu}$

Lorelei A Mucci

SC Mueller

Wolfgang Mueller-Klieser

Kenneth Muir

Colin R Muirhead

Hasan Mukhtar

Clive Mulatero

Charles G Mullighan

Alastair John Munro

Rajmohan Murali

Masaaki Muramatsu

Peter Murchie

Susan K Murphy

Graeme Murray

Iain Murray

Liam J Murray

David G Mutch

Luciano Mutti

Jeff Myers

Paivi Myllynen

Ivan R Nabi

Christina M Nagle

Raj Naik

MS Nai

Norifumi Naka

Masayuki Nakagawa

Yasutomo Nakai

Takuro Nakamura

Naoki Nakaya

Harikrishna Nakshatri

Josephine Nalbantoglu

Joo-Hyun Nam

Arlene Naranjo

Ghoutham Narla

Steven A Narod

A Nath

Katherine Nathanson

Steffan T Nawrocki

Beatrice Nawrocki-Raby

Florence Ndikum-Moffor

Richard D Neal

Andrea Necchi

Sarah Nechuta

Sattva S Neelapu

Albrecht Neesse

Brad Nelson

Charles B Nemeroff

John Neoptolemos

Kirsten Nes

Dirk M Nettelbeck

Alfred I Neugut

Nicole S Nevadunsky

Peter A Newman

Wai-Tong Ng

Samuel Ngan

Paul L Nguyen

Hazel Nichols

Larry Nichols

Nicola Nicolai

Carsten Nieder

Gunter Niegisch

Torsten O Nielsen

M Angela Nieto

Takashi Niizeki

Hans Nijman

Katharina Nimptsch

Reiko Nishihara

N Nitin

Donato Nitti

O Nkomazana

Makoto Noda

Leticia Nogueira

Anju Nohria

Franco Nole

Bernard Nordlinger

Mette Norgaard

Anna Novarino

Ariela Noy

Bernd Nuernberg

Ula Nur

Maureen O'Brien

Kenneth J O'Byrne

Anne O'Donnell

Norma O'Donovan

Patrick O'Dwyer

Ruth M O'Regan

Noelle O'Rourke

Joyce O'Shaughnessy

Jacintha O'Sullivan

Kjell Oberg

Kazuhiko Ogawa

Shuji Ogino 
Mala Pande

Herbert Pang

David J Panka

Lewis Pannel

Liron Pantanowitz

Klaus Pantel

V Papadimitrakopoulou

Konstantinos Papazisis

Carole A Parent

Fabrizio Parente

Carol Parise

Jin Young Park

Kevin Park

Sook Ryun Park

Sue K Park

Yoon Jung Park

Young Kyu Park

Alexander Parker

Anne Parker

Donald Maxwell Parkin

Maurizio Parola

Paola Parrella

Matthew Pase

Francesca Pasi

Maria Pasic

Elena B Pasquale

Giuseppe Pasqualetti

J-M Pasquet

Harvey I Pass

Uday Patel

Gaurav Pathria

Sujata Patil

Santosh Kumar Patnaik

Julietta Patnick

Ruth E Patterson

Jim Paul

Torbjorn Paulsen

Nicholas Pavlidis

David Pawe

Raheem J Paxton

Katherine Payne

Richard J Payne

Mick Peake

Praveen Peddu

Marc Peeters

Miguel A Peinado

Yuri Pekarsky

Giuseppe Pelosi

Paivi Peltomaki

Claudio Pelucchi

Barbara Pence

Nicolas Penel

Hanxiang Peng

Trevor Penning

George Pentheroudakis

George Penthroudakis

Kay Pepin

P Perego

Giovanni Perini

Neil D Perkins

Sven Perner

Francesco Perrone

Antoinette Perry

Rajendra Persad

Godefridus J Peters

$S$ Peters

SH Petersen

George Petheroudakis

Julian Peto

Fausto Petrelli

Edgar Petru

Salvatore Petta

Russell D Petty

Carmel J Pezaro

Raffaele Pezzilli

Georg Pfeiler

Giao Phan

Paul Pharoah

Joanna Phillips

Wayne A Phillips

Amanda I Phipps

Jenny Phipps

Didier Picard

Flavia Pichiorri

Martin Pichler
Joel Picus

Elizabeth Pienaar

John Pierce

Jean-Yves Pierga

Marco Pierotti

Arwen H Pieterse

Filippo Pietrantonio

Sandro Pignata

Ymera Pignochino

Albrecht Piiper

Roberto Pili

Paul F Pinsky

R Pinto-Leite

Kirstin Pirie

Giuseppe Pirozzi

Paola Pisani

Marie Plante

Ruben R Plentz

Elizabeth Ruth Plummer

Julien Poissy

Katja Pokrovskaja Tamm

Jerry Polesel

D Brent Polk

Jeffrey W Pollard

Rodney F Pommier

Alfonso Pompella

Martin G Pomper

Selvarangan Ponnazhagan

Elizabeth Poole

Helena Populo

Emilio Porfiri

Camillo Porta

Don W Powell

Helen A Powell

James Powell

Setia Praman

Guy Pratt

Jochen HM Prehn

Hans Prenen

David Press

Jean-Luc Pretet

Allan Price

Pat Price

Timothy J Price

John N Primrose

J Howard Pringle

Catrin Pritchard

Kathy Pritchard-Jones

Giuseppe Procopio

David Propper

Mark Puder

Fabio Puglisi

Rosa Puigpinos

Eero Pukkala

Lorenza Putignani

C Querfeld

Denis Querleu

Corinne Quittau-Prevostel

Andrew Quon

Akram F Qutob

Franz Rodel

Samuel Rabkin

Sylvia Rabstein

John A Radford

James M Rae

Arash Rafii

Estelle Rage

Derek Raghavan

Derek Raghavan

Antonella Ragnini-Wilson

Nuh Rahbari

Rakhshanda Layeequr Rahman

NJH Raijmaker

Sara Raimondi

Ganesh Raj

P Rajaraman

AK Rajasekaran

John K Ramage

Santiago Ramon y Cajal

Ajay Rana

Sheela Rao

Bernardo L Rapoport

Kakil Ibrahim Rasul

Elena S Ratner

Farhad Ravandi
Dipankar Ray

Eric Raymond

Clare JM Reade

Christopher J Recklitis

Nicholas Simon Reed

Katherine Reed-Hayes

Myrddin Rees

Helen Reeves

Meredith M Regan

David Rehkopf

Peter Reichard

Alison Reid

Mary Reilly

Celso Reis

Jorge S Reis-Filho

Paul L Reiter

Russel J Reiter

Jordi Remon

Andrew G Renehan

Michele Reni

Edyta Reszka

James M Reuben

Miriam Reuschenbach

Brent Rexer

John V Reynolds

Peggy Reynolds

Antoni Ribas

Benjamin Ribba

Daniel Araki Ribeiro

Treva K Rice

Frances M Richards

Angela M Riddell

David J Riese II

Brian I Rini

Michael Rink

Olivier Rixe

Naiyer A Rizvi

Ivana Rizzuto

WH Roa

Lukas Rob

Kathryn Robb

$\mathrm{K}$ Thomas Robbins

Karin Roberg

acques Robert

Lidia Robert

Ashley Roberts

Thomas M Roberts

Matthew K Robinson

Stephen Robinson

Craig N Robson

Rodney P Rocconi

Christoph Rocken

Andrew W Roddam

Sjoerd Rodenhuis

G Rodin

George Rodrigues

Claire Rodriguez-Lafrasse

Jong-Lyel Roh

Mi Ryung Roh

Sergio Roman-Roman

Hanna Romanska

Roberto Ronca

Cecile M Ronckers

Igor B Roninson

Roberta Rosa

Juan A Rosado

Joseph Roscoe

Rafael Rosell

Mark Rosen

Lynn Rosenberg

Karin A Rosenblatt

Calvin Roskelley

Bernard Rosner

Paul J Ross

Richard Ross

Charles Rosser

Marta Rossi

Stefano Rosso

Charlotte Rotbol Boje

Ana Rouzaut

Brian G Rowan

Clare Rowntree

Campbell SD Roxburgh

Pradip Roy-Burman

Linda Rubenstein

Kathryn J Ruddy

Michelle A Rudek

Anja Rudolph

Elena Rugali

Simon Rule

Valerie W Rusch

Stuart D Russell

Hege Elisabeth Giercksky Russnes

Antonio Russo

Vincenzo C Russo

Gordon JS Rustin

Sergio Rutella

Mark J Rutherford

Sascha Rutz

Anderson J Ryan

Lisa Ryden

Steve Ryder

Anne M Ryhanen

Ricardo Sanchez-Prieto

Henrik Toft Sorensen

Everardo D Saad

Fred Saad

Fahri Saatcioglu

Renaud Sabatier

Stephen Safe

Satoru Sagae

Hege Sagstuen Haugnes

Kamal Saini

A Sakamoto

R Sakata

Antonio Salar

Ramon Salazar

Flavio Salazar-Onfray

Mohammad Saleem 
Manuela Schmidinger Kirsten Tryde Schmidt Marjanka K Schmidt

Susanne Schmidt

Fernando Schmitt

Hans-Joachim Schmoll

Carole M Schneider

Patrick Schoeffski

Sara J Schonfeld

Leo Schouten

Heiner Schrewe

Roland Schueler

Patrick J Schuler

Fabio Schutz

Joachim Schuz

Carolyn E Schwartz

Richard C Schwartz

Judith Ann Schwartzbaum

Kathryn Schwertfeger

Jean-Paul Sculier

Susan Searles Nielsen

David Sebag-Montefiore

Veronika Seebacher

Rosalind Segal

Astrid Sehested

Andrew Seidman

Jeffrey D Seidman

Arnaud Seigneurin

Naohiko Seki

Ikuo Sekine

Galina Selivanova

Karri Seppa

Natalie Serkova

Mukund Seshadri

Cristiana Sessa

Ivana Sestak

Gautam Sethi

Takashi Seto

Veronika Sex

Thomas N Seyfried

CMJC Seynaeve

Alessandro Sgambato

Kashif Shafique

Jonathan Shamash

Lalitha K Shankar

Rong Shao

David J Shapiro

Rohini Sharma

Sherven Sharma

Richard Sharpe

Jacqueline A Shaw

Paul HS Shaw

Fatma M Shebl

John A Shepherd

Neil A Shepherd

Linda A Sherman

Qian Shi

Yufang Shi

MS Shiels

Katsumi Shigemura

Xianglin Shih

Hiroshi Shiku

Takaya Shimura

Sandra J Shin

Young Kee Shin

Nobuo Shinohara

Akiko Shiotani

Janet Shipley

Shiri Shkedi

Maya Shmulevitz

Young Kee Shong

Susan Short

Timothy N Showalter

Vijayalakshmi Shridhar

Rachna Shroff

Michael Shurin

Oliver Sieber

Jill M Siegfried

Weiva Sieh

Sabina Sieri

Anieta M Sieuwerts

Andrew G Sikora

Andrew Silver

Sarah C Sim

Edgar Simard
Alice E Simon

Ken Simpson

Susanne Singer

$\mathrm{H}$ Singh

Pankaj Singh

Siddharth Singh

Alphonse E Sirica

Freddy Sitas

Stephanie Siu

Anita Sjolander

Arne Skerra

Roderick Skinner

Klaudia Skrzypek

Ira Skvortsova

George W Sledge, Jr

Jonathan Sleeman

Brian M Slomovitz

Susan R Slovin

Giedre Smailyte

David P Smith

Laura Smith

Mark Smyth

Xavier Soberon

Riccardo Soffietti

Jerome solassol

Tora S Solheim

Hatem Soliman

Keith R Soloman

Navita Somaiah

Ji Woong Son

Erwei Song

Mingyang Song

Stephen T Sonis

Guru Sonpavde

Patsy Soon

Jean-Charles Soria

Jorma Sormunen

Robert Soslow

John Souglakos

Jennifer Southgate

Rhonda F Souza

Teresa Spadea

Paul N Span

Joseph A Sparano

Anna Spathis

David Spencer

James F Spicer

Philippe EE Spiess

Alexander Spira

Yves St-Pierre

Cathy Staedel

John Staffurth

Lukas JA Stalpers

Ronald L Stam

Gordon Stamp

Andreas Stang

Giorgio Stanta

Martin Stanulla

Anastasios Stathis

Eike Staub

Justin Stebbing

Robert JC Steele

Christopher B Steer

Arnulf Stenzl

Julie Sterling

Neil M Steven

Richard G Stevens

William P Steward

Colin J Stewart

David J Stewart

Friedrich Stiefel

Stephen Stilgenbauer

Christian-Martin Stock

Martin R Stockler

Patrick C Stone

Angela Stover

Dirk Strauss

Jonathan Strefford

Staffan Stromblad

Kevin Struhl

Nick Stuart

Urs E Studer

Susan R Sturgeon

Erich M Sturgis

Xiaoping Su
Vivek Subbiah

Yoshiyuki Suehara

Richard Sullivan

Ryan Sullivan

Katherine Sumpter

Shi-Yong Sun

Xiankai Sun

Sudha Sundar

Karin Sundstrom

Joseph JY Sung

Lillian Sung

Hiromu Suzuki

Asgerdur Sverrisdottir

Marek Svoboda

Kristin R Swanson

Colleen Sweeney

Anthony J Swerdlow

$\mathrm{R}$ Paul Symonds

Janos Szollosi

Attila Szabo

Zoltan Szallasi

Anne Szarewski

Peter Szlosarek

Mario Sznol

Alphonse G Taghian

Pierosandro Tagliaferri

David Taieb

Emanuela Taioli

Brian D Tait

Diana Tait

Nobuyuki Takakura

iwamoto takayuki

Koji Takeda

Shinsuke Takeno

Kengo Takeuchi

Evelyn O Talbott

H Tamai

Nguan Soon Tan

Shinji Tanaka

Shinya Tanaka

Torgrim Tandstad

Chih-Hsin Tang

Damu Tang

A Tangoku

Yusuke Tanigawara

Ian F Tannock

YX Tao

Ahmad A Tarhini

Eric Tartour

Paul Ian Tartter

Kristin Austlid Tasken

Elena Tassi

Martin HN Tattersall

Christoph Tausch

Alessandra Tavani

Catherine Taylor

Robin Taylor Wilson

Muy-Teck Teh

Jose M Teixeira

Arnoud Templeton

Patricia Tenni

Paul D Terry

Leon WMM Terstappen

Andrew Teschendorff

Ugo Testa

Chris Barna Teszler

Mark Thalgott

GN Thalmann

Stamatios Theocharis

Anne CM Thiebaut

Calvin Thigpen

Victor LJL Thijssen

Robert Thimme

Chrissie Thirlwell

Fiona Thistlethwaite

Anish Thomas

Anne Thomas

Gareth J Thoma

Deborah Thompson

Deborah L Thompson

Erik W Thompson

John A Thompson

CA Thomson

Mangesh Thorat

Katherine Thornton

Melissa M Thrall

Aaron P Thrift

Lau Caspar Thygesen

C Tian

J Tian

Gao Tianwen

Raoul Tibes

Jim Tiernan

Madeleine MA Tilanus-Linthorst

Jozsef Timar

Albert Tiong

Marcello Tiseo

Linda J Titus

Katherine HR Tkaczuk

Giuseppe Toffol

Philip J Tofilon

Masakazu Toi

Anthony W Tolcher

Trygve Tollefsbol

Rob AEM Tollenaar

Bertrand Tombal

Giuseppe Tonini

Jeff Toretsky

Adetunji T Toriola

Luigi Tornillo

Jorge R Toro

Keila E Torres

Marie Louise Torring

Giampaolo Tortora

Victoria Tovar

Philip C Trackman

Tiffany A Train

Nham Tran

Gerd Trano

Lois B Travis 
Brian G Van Ness

Nicolien van Ravesteyn

HM Van Santen

Julia J van Tol-Geerdink

MATM van Vugt

Carter van Waes

Bart Vanhaesebroeck

Sheelu Varghese

Vasilis K Vasiliou

C Vaske

Erik Vassella

Thomas Vaughan

Jean-Nicolas Vauthey

Andrea Vecchione

Peter Vedsted

Alfredo Vellido

Julio Vera

Alain Vergnenegre

Henk MW Verheul

C Verhoef

Helena M Verkooijen

Sally Vernon

Michael M Vickers

Erica Villa

Judith Grob Villablanca

Vincent Vinh-Hung

Yana Vinogradova

Jesus Vioque

Tapio Visakorpi

Tuomo Visuri

Umberto Vitolo

Emile E Voest

Bjorn G Volkmer

Andre $\mathrm{O}$ von Bueren

My von Euler-Chelpin

Daniel von Hoff

Srinivas Vourganti

Jana Vranova

Alina Vrieling

Sholom Wacholder

Madhuri Wadehra

Jon Wadsley

Anna-Dorothea Wagner

Simon Wagner

Richard Wakeford

Neal Walker

CR Walkley

Lucy Wall

Jo Waller

Paul M Walsh

Tom Walsh

Sarah Walters

Reinhard Walther

Jun Wan

Hans H Wandal
Fengfei Wang

Hongbing Wang

Jianliu Wang

Jianxiang Wang

Michael Wang

Tian Wang

Timothy Wang

Yaning Wang

Yaohe Wang

Yu Wang

Zhen Wang

Zhidong Wang

James E Ward

Padraig Richard Warde

Jane Wardle

Saman Warnakulasuriya

David Warr

Sudha Warrier

Lauren A Waters

Ashita Waterston

David I Watson

Joanna Watson

Christopher D Watt

Andrew Webb

Frank Weber

Ulrich Wedding

Joseph Tien-Seng Wee

Claire Weekley

Wilko Weichert

Ralph Weichselbaum

Zhang Weihua

Stephanie J Weinstein

SJ Weiss

James W Wells

Mary Wells

Jozefa Wesierska-Gadek

Andrew Westwell

Tim Whelan

Craig White

Jeff D White

Penny F Whiting

Stefan Wiehr

Stephen J Wigmore

Jenny Wilding

Karen Willard-Gallo

Gareth Haydn Williams

Stephen K Williamson

Kathryn M Wilson

Klas G Wiman

Thomas Winder

Eric Winquist

Ilka Wittig

Julie Anna Wolfson

Barbara Wollenberg

Cesar Wong
Kwong-Kwok Wong

Otto Wong

Sandra L Wong

Laurence Wood

Ian Woolhouse

Frank Worden

An Wouters

Jennifer Woyach

Ellen Wright

Penny Wright

Gang Wu

QiJun Wu

Wenting $\mathrm{Wu}$

Xiaosheng $\mathrm{Wu}$

Xifeng Wu

Au Wy

Luc Xerri

Hongping Xia

Liang Ping Xia

Yun-Fei Xia

Huasheng Xiao

Mang Xiao

Dan Xie

W Xie

Ren Xu

Yan $\mathrm{Xu}$

GL Xua

Steven Yale

T Yamasaki

Hiroko Yamashita

Hiroki Yamaue

Hai Yan

Chi-Chiang Yang

Dongyan Yang

HP Yang

TienYu Owen Yang

Tokujiro Yano

Mike Yao

Wendell G Yarbrough

Paul Yaswen

Weimin Ye

Douglas Yee

Edward TH Yeh

Sriram Yennurajalingam

Winnie Yeo

Kam C Yeung

Sai-Ching J Yeung

Min Yi

Chu-Yiu Yiu

Jun Yokota

Atsuko Yoneda

Harry Yoon

Kazuhiro Yoshida

Hideyuki Yoshitomi
Hye Jin You

Jianxin You

Mamoun Younes

Jane Younger

Tallal Younis

George M Yousef

Dominic $\mathrm{Yu}$

Jau-Song Yu

Kelly J Yu

Long $\mathrm{Yu}$

Shiying Yu

Jian Yuan

Yasuhito Yuasa

Wei Yue

Aziz Zaanan

Nadia Zaffaroni

Ian S Zagon

Flora Zagouri

Rosnah B Zain

John Raymond Zalcberg

G Zalcman

Raul Zamora-Ros

Marjolein Zanders

Paolo Zanotti-Fregonara

Marco Zappa

Paul Zarogoulidis

Hajo Zeeb

Pascal Zehnder

Laurent Zelek

Jason A Zell

Binhao Zhang

Gao Zhang

Jingsong Zhang

Wei Zhang

Wu Zhang

Yixian Zhang

Zhaolei Zhang

Shu Zheng

Wenxin Zheng

Binhua P Zhou

Ming Zhou

Ping-Kun Zhou

Jin Liang Zhu

Wei-Guo Zhu

Lucy Ziegler

Christoph C Zielinski

Richard Zigeuner

Inti Zlobec

Xiangyun Zong

Kathryn C Zoon

Manuel Zorzi

Istvan Zupko

Amado J Zurita-Saavedra 